\title{
Comorbid anxiety disorder has a protective effect in conduct disorder
}

\section{By Dr. Jessica K Edwards}

The presence of comorbid anxiety disorders (ADs) counteracts the effects of conduct disorder (CD) on facial emotion recognition, according to new research by Roxana Short and colleagues. In their 2016 study, the researchers compared the abilities of adolescents aged 12-18 years with $C D(n=28), A D(n=23)$, co-occurring $C D$ with $A D$ $(n=20)$ and typically developing controls $(n=28)$ in recognising various emotions (anger, fear, happiness, sadness and disgust) in images of faces representing different levels of emotional intensity.

They found that adolescents with CD had a generalised impairment in emotion recognition compared to the other two groups, but this may have been mediated by group differences in IQ. By contrast, adolescents with AD alone showed increased sensitivity to low-intensity happiness, disgust and sadness, indicative of an enhanced performance over the other two groups. Most interestingly, the comorbid AD plus CD group showed a similar overall performance in facial emotion recognition ability as the control group. Based on these findings, the researchers propose that $A D$ has a potentially protective role in $C D$, and that targeted interventions, such as emotion training, may be more effective in those with $C D$ alone than those with comorbid AD.

Further research is now needed to examine the contribution of IQ and gender to these described effects.

\section{Referring to:}

Short, R.M.L., Sonuga-Barke, E.J.S., Adams, W.J. and Fairchild, G. (2016), Does comorbid anxiety counteract emotion recognition deficits in conduct disorder? J. Child. Pscyhol. Psychiatr. 57: 917-926. doi: 10.1111/ jcpp.12544.

\section{Glossary:}

Conduct disorder (CD): $C D$ is characterized by behaviour that violates either the rights of others or major societal norms. To be diagnosed with conduct disorder, symptoms must cause significant impairment in social, academic or occupational functioning. The disorder is typically diagnosed prior to adulthood. 